\title{
Limitations of wind power availability over Europe: a conceptual study
}

\author{
P. Kiss ${ }^{1}$ and I. M. Jánosi ${ }^{1,2}$ \\ ${ }^{1}$ Department of Physics of Complex Systems, Roland Eötvös University, Pázmány Péter sétány 1, 1117 Budapest, Hungary \\ ${ }^{2}$ Institute for Mathematics and its Applications, University of Minnesota, 207 Church Street SE, Minneapolis, \\ MN 55 455, USA
}

Received: 2 June 2008 - Revised: 14 August 2008 - Accepted: 12 September 2008 - Published: 3 November 2008

\begin{abstract}
Wind field statistics are evaluated from the ERA-40 data bank covering a period of 44 years with a temporal resolution of $6 \mathrm{~h}$. Instantaneous wind speed values are provided in geographic cells of size $1^{\circ} \times 1^{\circ}$ (lat/long) for surface $(10 \mathrm{~m})$ and $1000 \mathrm{hPa}$ pressure heights. Potential wind power generation is estimated in two steps. Firstly, the wind speed at hub height is approximated from surface data based on the statistical analysis of the wind and geopotential records for $1000 \mathrm{hPa}$ pressure level. Secondly, the wind speed values are transformed by an idealised power curve fitted for measured data. The model time series are fed into various hypothetical electric networks. The main quantity of interest is the aggregated output from the networks. A reference power time series is determined for a static network connecting each continental site and an envelope of $1^{\circ}$ around the shorelines (representing off-shore locations) over Europe. This time series exhibits a low average value and a marked annual periodicity. Wind power integration over limited areas results in higher average outputs at the expense of stronger fluctuations. The long-range spatial correlations of the wind field limit the level of fluctuations strongly which can not be eliminated either by an increase of the area of integration or by dynamic control. This study is fully conceptual, however it demonstrates the limitations of wind power integration over Europe.
\end{abstract}

\section{Introduction}

It is well known that wind is a highly volatile resource, nevertheless the growth of worldwide installed wind power capacity is almost exponential. European leaders signed up in March 2007 to a binding EU-wide objective to supply 20\% of their energy needs from renewable sources such as biomass, hydro, wind and solar power by 2020 . The next task is to define specific national targets to achieve the overall figure, taking into account each country's potential in determining an optimal resource portfolio.

Wind power scepticism is mostly based on the fact that energy supply is often not available from any single cluster of turbines. Contrarily, proponents argue that "wind always blows somewhere", thus energy generation integrated over a large area is less variable than generation from a single region. This statement is supported by several studies (Landberg, 1997; Ackermann and Söder, 2002; Holttinen, 2005; Giebel, 2007), nevertheless it is also a fact that the medium range (hours to days) variability can not be decreased by distributed generation as effectively as the short range (seconds to minutes) fluctuations (Ackermann and Söder, 2002). The integration of electricity production over increasing areas has natural barriers. At present, wind turbine installation in EU countries is confined to the continent and an offshore belt, lowered by urban areas, various land-use functions (e.g., bioreserves) and mountain regions of high elevation. Assessment studies over Europe (Landberg, 1997; Giebel, 2007) analysed the main aspects of large scale distributed wind power production. Giebel (2007) modeled the whole electrical generation system of Europe with varying penetrations of wind power. The main conclusion was that wind energy can contribute more than $20 \%$ of the European demand without significant changes in the system and can replace conventional sources worth about $10 \%$ of the installed wind power capacity. 
In order to achieve a substantial penetration of distributed energy resources, it is necessary to address the key issues related to their integration into existing and future energy systems. One of the most important challenges seems to be the management of fluctuations in the electricity production from renewable energy sources (Lund, 2005). The incorporation of higher levels of stochastic generation requires the development of "horizontally operated" power systems (Papaefthymiou et al., 2006), where dispatchable generation units adapt to the variations of the non-dispatchable ones. Traditional power systems based on thermal plants have limited wind power acceptance capacity constrained by an adequate level of system stability (Katsaprakakis et al., 2007). However, the development toward the integration of distributed generation is continuous, including a number of new technologies such as intelligent switches, adaptive protection, or web based information and control systems (Dondi et al., 2002; Pepermans et al., 2005).

A recent comprehensive analysis by Archer and Jacobson (2005) concluded that the global wind power potential is huge (five times the world's total energy demand in the year 2000), but several practical barriers need to be overcome to realise this potential. In this work we intend to contribute to a better characterisation of the constraints arising from the statistical properties of wind field over Europe. As a reference, we construct model time series for wind power based on ERA-40 re-analysis (Uppala et al., 2005) wind data covering 44 whole years and the geographic area of the continent and the surrounding maritime regions. We test different architectures of "perfect" hypothetical grid configurations, where all the generated electricity is integrated without any technical restrictions. This approach is certainly far from any technical actuality, however it helps to demonstrate the unavoidable limitations coming from the intermittent properties and spatiotemporal correlations of wind. We show that the large wind power variability can not be eliminated even by integrating over the whole continent. Of course, we do not question the overall usefulness of wind energy utilisation, nevertheless an optimal exploitation can not be realised without the consideration of existing physical constraints. We are fully aware of the fact that damping of variability of wind energy supply will have to be achieved by integration with complementary renewable sources (such as solar or biomass), however planning of future energy systems is not possible without a proper characterisation of the individual resources.

\section{Data and methods}

We evaluated ECMWF's ERA-40 re-analysis data (Uppala et al., 2005) consisting of the $u$ (eastward) and $v$ (northward) orthogonal components of the horizontal wind field at $10 \mathrm{~m}$ above ground and at the $1000 \mathrm{hPa}$ pressure level, together with the $1000 \mathrm{hPa}$ geopotential, for a time period of 44 years between 1 September 1958 and 31 August 2002. Four instantaneous values are available each day for synoptic hours 00:00, 06:00, 12:00 and 18:00 UTC at each geographic location. The spatial resolution is $1^{\circ} \times 1^{\circ}$ (lat/long), and a given value for an atmospheric variable is considered to be representative for the whole cell. The representation of sub-scale events are not attempted. Our analysis is restricted to a geographic area covering Europe between $35^{\circ} \mathrm{N}-75^{\circ} \mathrm{N}$ latitude and $20^{\circ} \mathrm{W}-40^{\circ} \mathrm{E}$ longitude. Several statistical characteristics for the surface $(10 \mathrm{~m})$ wind speed $s=\sqrt{u^{2}+v^{2}}$ are extracted for the same data set in Kiss and Jánosi (2008). The validation of the data bank is out of the scope of this work, however we note that wind measurements are among the most fundamental input variables of the re-analysis procedure (Uppala et al., 2005).

We note however that the limited data resolution makes this study rather conceptual. Good sites for wind power are often characterised by local features that channel the winds, such as topography ridges, canyons, or strong horizontal thermal gradients. Such features are mostly unresolved in the ERA-40 dataset, therefore wind speed estimates are probably biased toward lower values.

\subsection{Wind power estimation from surface wind speed data}

Wind speeds measured at $10 \mathrm{~m}$ frequently differ from speeds at potential turbine hub heights $(60-120 \mathrm{~m})$, therefore tower measurement for (at least) one year has became an industrial standard for plant siting (Burton et al., 2001; EWEA, 2004). The wind shear is known to depend on stratification, stability, vertical heat flux or wind direction over complicated topography (Garratt, 1994). High resolution wind profile records are not available in the ERA-40 data bank, therefore we implemented a simple statistical procedure for wind speed estimates at higher than $10 \mathrm{~m}$ levels.

The geopotential for a fixed pressure level is essentially the height (above mean sea level), where the pressure has the given value, multiplied by the standard gravity at mean sea level. The re-analysis provides continuous meteorological fields by extrapolations, thus the altering $1000 \mathrm{hPa}$ geopotential height $h$ often exhibits negative values, especially over high geographic elevations (see Fig. 1). In fact, data scarcity demanded also spatial averaging over the continent in order to extract an approximate average wind profile based on the speed ratio $s_{h} / s_{10}$ for $h>10 \mathrm{~m}$. The results are shown in Fig. 2. At a given bin of $1000 \mathrm{hPa}$ pressure height $h \pm 2.5 \mathrm{~m}$, the speed ratio histograms exhibit well defined mode (most probable) values (Fig. 2a). The mode profile (Fig. 2b) can not be perfectly fitted either by the logarithmic (Tennekes, 1973; Carl et al., 1973) or the power-law (Hsu et al., 1994; Farrugia, 2003; Sedefian, 1980) assumptions used widely in the literature. Consequently, we introduced the simplest possible estimate based on the available data which is the multiplication of instantaneous wind speeds at $10 \mathrm{~m}$ by a factor of 1.28 . 

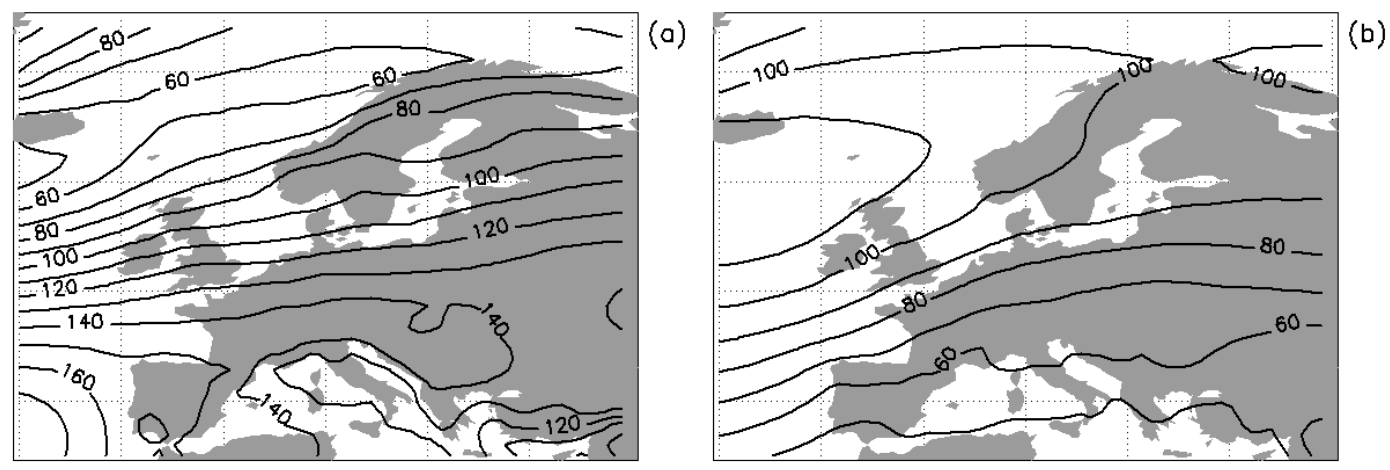

Fig. 1. (a) Average value, and (b) standard deviation for the $1000 \mathrm{hPa}$ geopotential height above sea level for the 44 years covered by the ERA-40 data bank. Contour lines are drawn with a spacing of $10 \mathrm{~m}$, see the labels.
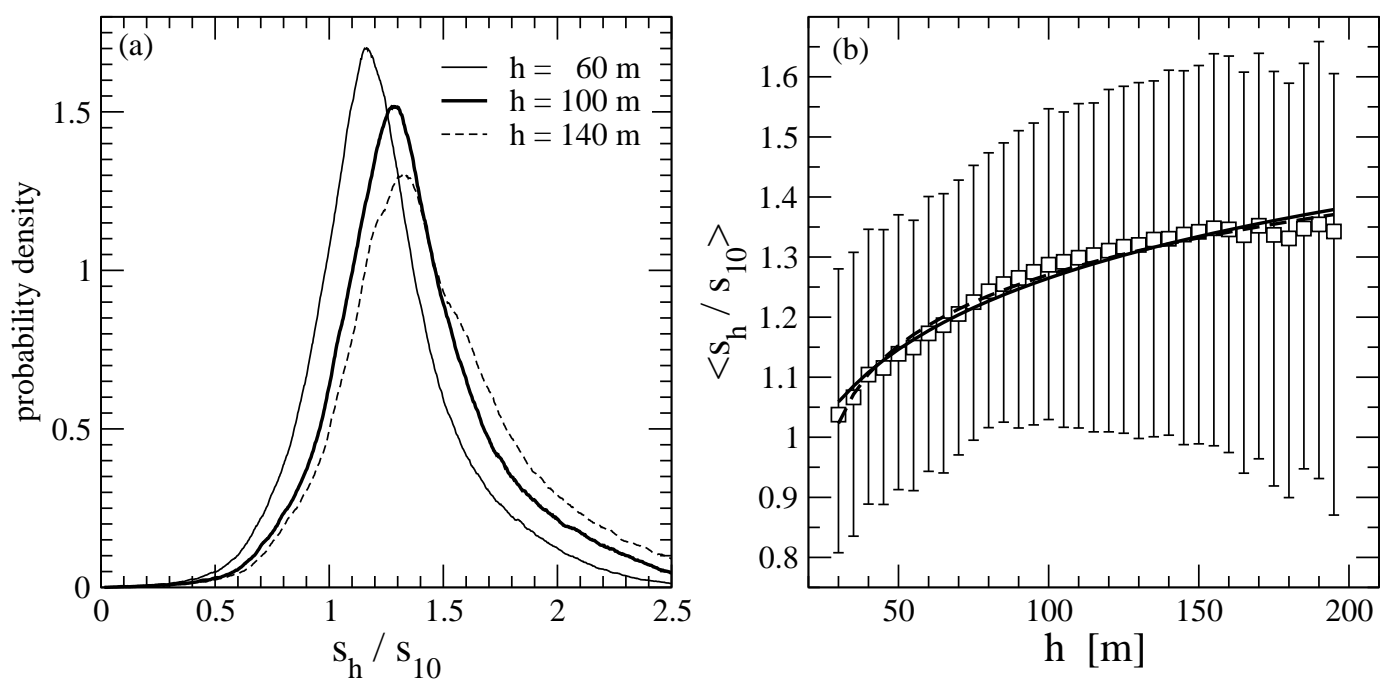

Fig. 2. (a) Normalised histogram of wind speed ratio $s_{h} / s_{10}$ for three different bins $h \pm 2.5 \mathrm{~m}$ (see labels), where $h$ is the mean $1000 \mathrm{hPa}$ pressure height above ground. The histograms contain aggregated data over the continental geographic area (see Fig. 4). (b) The mode of wind speed ratio profile as a function of $1000 \mathrm{hPa}$ pressure height $h$ (white symbols). The error bars indicate the half width of histograms shown in (a)). Solid line is a logarithmic fit, dashed line illustrates a power-law with an exponent value of 0.10 .

This gives a crude measure of wind speed for $100 \mathrm{~m}$ over land and 100-130 m over sea surface. Different multipliers obviously change the numerical values for subsequent wind power estimates, but do not change the main conclusions.

The second step is to perform wind power estimates from the rescaled surface wind speed time series. The standard method for a given turbine is based on the empirical power curve (Burton et al., 2001) provided by the producers (see the power curves e.g., http://www.enercon.de, http:// www.nordex-online.com, http://www.vestas.com). The rated power can be very different for smaller and larger generators, however typical power curves share several other aspects (Fig. 3). The cut-in wind speed $s_{c i}$ is usually $3-5 \mathrm{~m} / \mathrm{s}$, then the curve exhibits a power-law range with an exponent value close to 3 . There is a crossover at around $s_{x} \approx 11-15 \mathrm{~m} / \mathrm{s}$ to a plateau (representing the rated power regime of active blade pitch control), and the cut-out wind speed $s_{c o}$ is $25 \mathrm{~m} / \mathrm{s}$ for most commercial constructions. An idealised power curve is depicted in Fig. 3, where the following form is used:

$$
\begin{array}{lll}
P(s)=a_{0}\left(s-s_{c i}\right)^{\beta} & \text { if } & s_{c i} \leq s \leq s_{X} \\
P(s)=\frac{a_{1}}{1+\exp \left[-(s-b)^{c}\right]} & \text { if } & s_{x}<s \leq s_{c o} \\
P(s)=0 \quad \text { if } \quad s<s_{c i} & \text { or } & s>s_{c o}
\end{array}
$$

The constant values $s_{c i}=1.2 \mathrm{~m} / \mathrm{s}, s_{x}=10.0 \mathrm{~m} / \mathrm{s}, s_{c o}=25.0 \mathrm{~m} / \mathrm{s}$, $\beta=2.79, \quad a_{0}=0.161, \quad a_{1}=100.0, \quad b=9.16$, and $c=1.05$ are empirical parameters obtained by fitting high resolution ( $\Delta t=10$ min with total duration of 3 years) measured data (nacelle anemometer readings with instantaneous power output) for two Enercon E-40 turbines (rated power $600 \mathrm{~kW}$ ) 


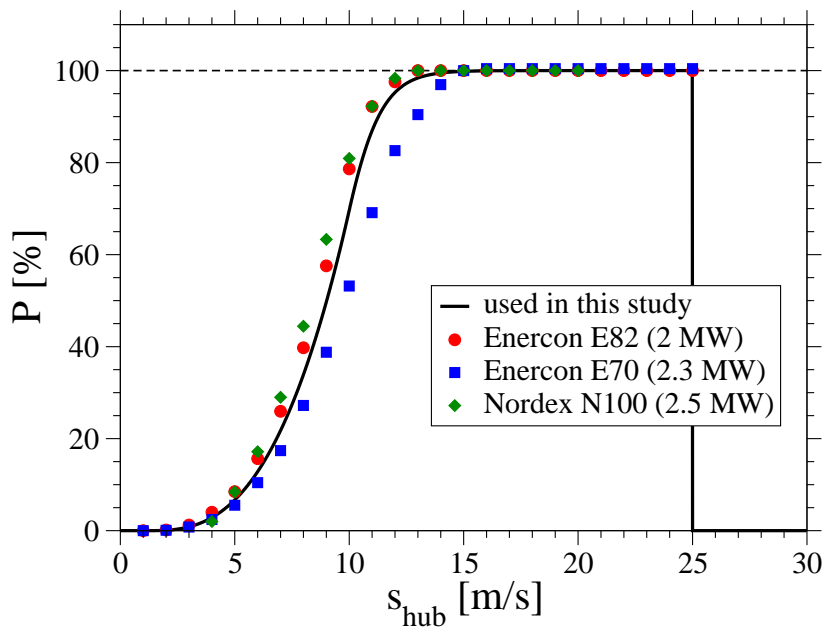

Fig. 3. Black line: Fitted power curve (percentage output power $P$ as a function of wind speed at hub height $s_{\text {hub }}$ ) for wind power estimates, see Eq. (1). The symbols indicate normalised power curves extracted from the product data sheets for three modern turbines (see legends).

installed near Mosonszolnok, Hungary. Normalisation is performed by the maximal power output instead of the rated power, because the former value often exceeds the latter by a few percents. The piecewise continuous form Eq. (1) provides a simple nonlinear filter to produce estimated wind power time series at each individual geographic location.

\section{Results}

We computed model time series (see Sect. 2) for the locations where wind turbines might be practically installed. Possible off-shore sites are taken into account by an envelope of $1^{\circ}(100-120 \mathrm{~km})$ around the coastlines (Iceland and the Faroe Islands are not considered), the total number of geographic cells is 1325 over this area. The long time mean value of estimated power exhibits a strongly uneven geographic distribution (Fig. 4a), but this is a rather well known fact (Elliott et al., 1986; Troen and Petersen, 1989; EWEA, 2004; Archer and Jacobson, 2005). The best locations for wind energy generation are off-shore sites near the Atlantic Ocean. Extended solid surfaces do not only moderate wind speeds but increase its variability over land (Fig. 4b).

Next we can imagine that different geographic cells are connected by a very efficient electric network, where fluctuations do not limit the integration of wind energy and there are no losses. This assumptions are far from reality, however our aim is to compare different ideal network architectures with the hope that a proper construction of electricity integration helps to eliminate the strong natural variability of the wind resource.
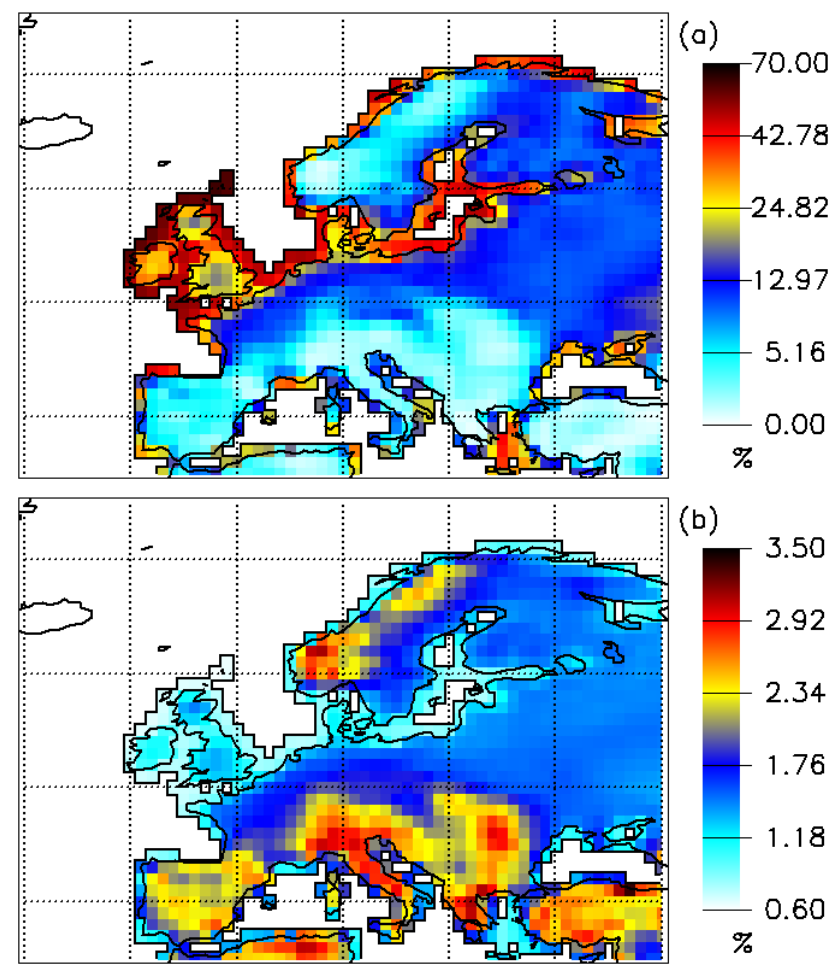

(b)

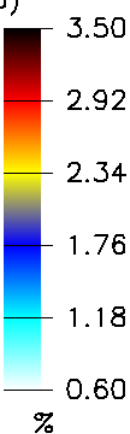

Fig. 4. (a) Average wind power in units of percentage capacity factor (logarithmic colour scale), and (b) coefficient of variation (standard deviation over average value) for each individual geographic cell and for the whole temporal period of 44 years (linear colour scale).

\subsection{Whole continent static integration of wind energy}

As for an appropriate reference, first we show the results for a static ideal network which integrates the instantaneous electric outputs for all of the 1325 geographic cells indicated in Fig. 4. Figure 5a shows the time series $P_{\text {tot }}(t)$ in units of aggregated capacity factor which is a convenient measure allowing any values for rated power at different locations. Here the rated power is distributed uniformly (cell size changes are not considered). The fluctuations are highly non-Gaussian (Fig. 5b), however we could not find a simple analytical form for an adequate fit. The long time mean value for the aggregated output is $13.9 \%$ (with a standard deviation of $6.6 \%$ ), which is considerably lower than the sustained average capacity factor at optimal off-shore sites (Pryor and Barthelmie, 2001; Henderson et al., 2003). The time series in Fig. 5a exhibits a marked annual periodicity and a weaker daily cycle revealed by the power spectrum in Fig. 5c. The aggregated output never drops to absolute zero, nevertheless it is less than $10 \%$ of the total rated power for 104-105 days in a year. It is also remarkable that the absolute minimum is as low as $1.1 \%$, and the absolute maximum does not exceed $51.3 \%$ 

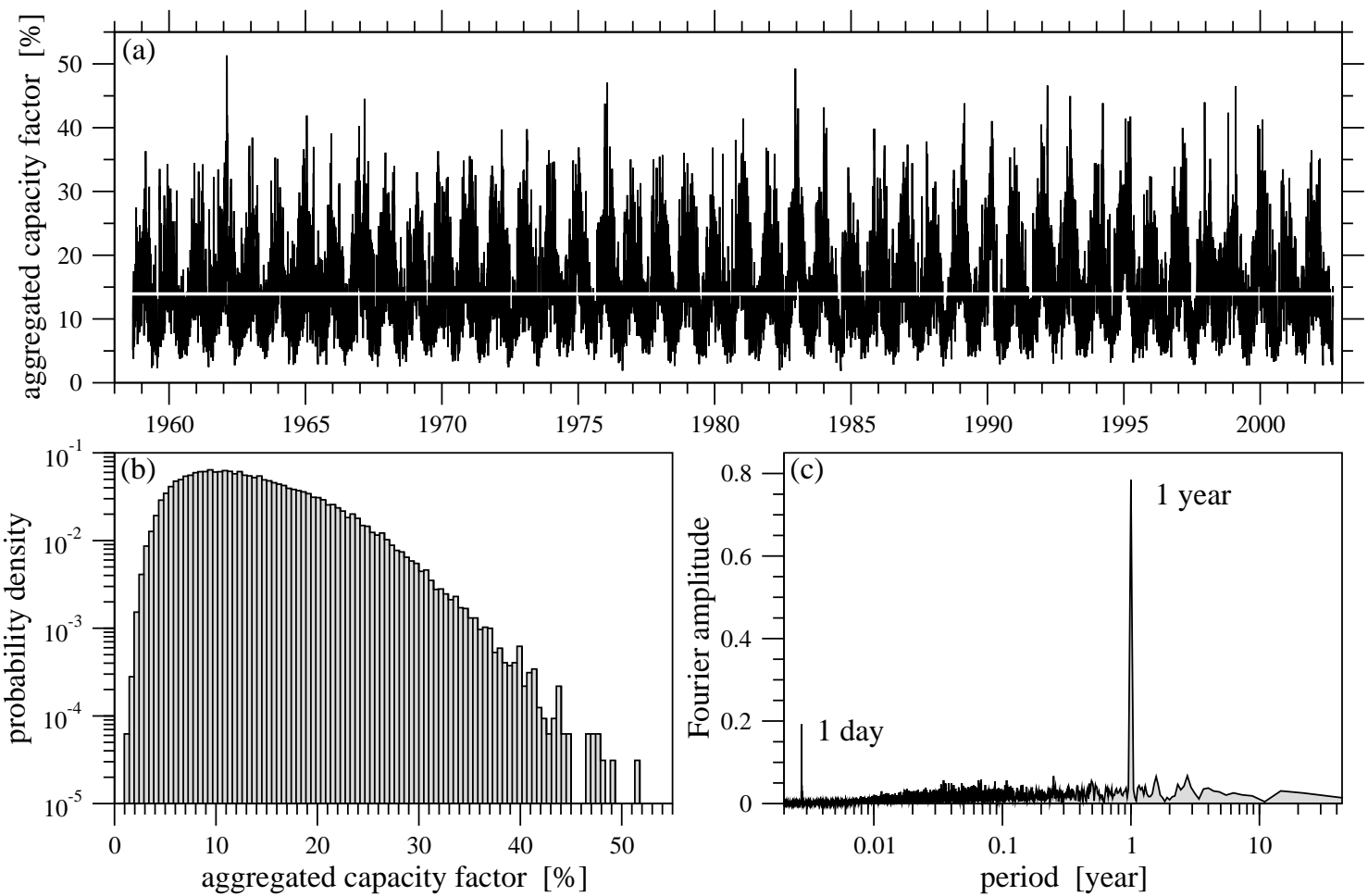

Fig. 5. (a) Time series of aggregated wind energy production over 1325 sites. The 44 year mean capacity factor is $13.9 \%$ (white line). (b) Normalised amplitude distribution of the time series. (The vertical scale is logarithmic.) (c) Power spectrum of the time series as a function of period. The two isolated peaks indicating a strong annual and a weaker daily periodicities are marked.
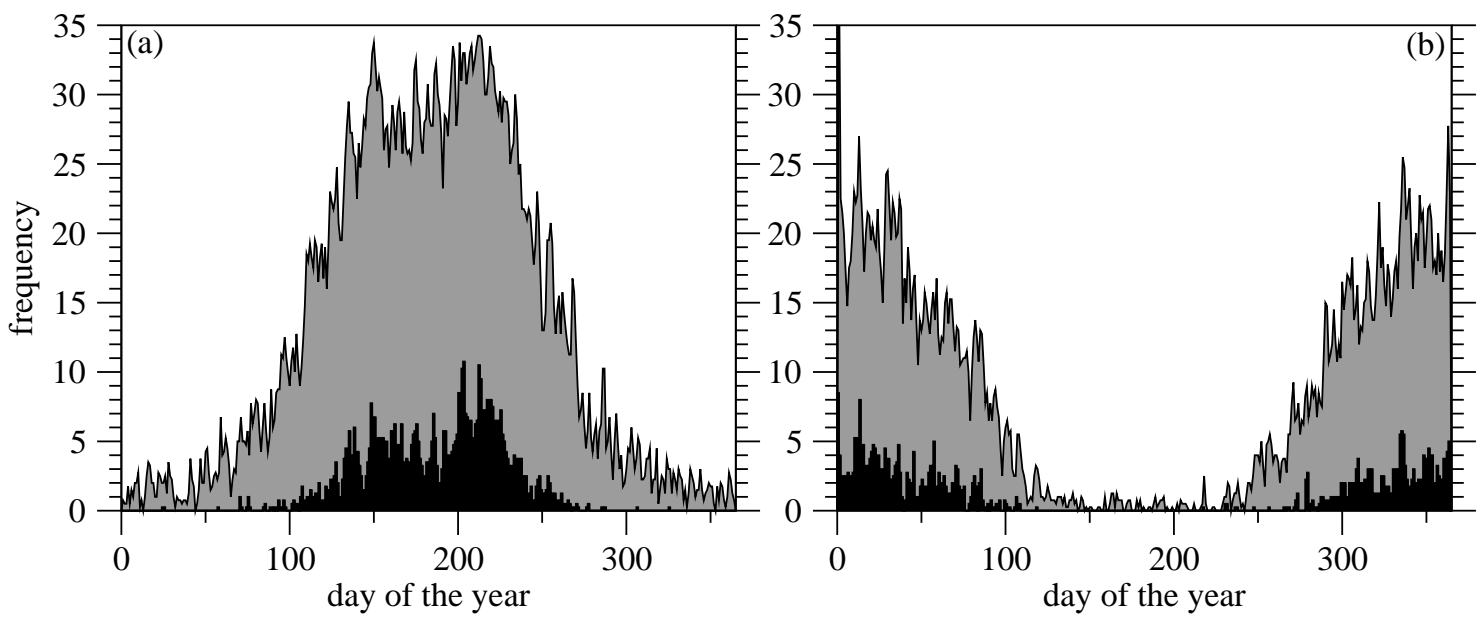

Fig. 6. (a) 44 year frequency of calendar days with less than $10 \%$ (gray) and less than $5 \%$ (black) aggregated output. (b) The same as (a) for days with more than $20 \%$ (gray) and more than $30 \%$ (black) output.

during the period of 44 years. These facts clearly indicate that a fully connected static network of uniform rated power distribution over the whole continental Europe is highly suboptimal with huge idle capacities and a marked seasonality.

Figure $5 \mathrm{c}$ might suggest that the strong annual periodicity could be exploited for long-time output scheduling. Figure 6 illustrates, however, that this is not really feasible. Signifi- cantly lower $\left(P_{\mathrm{tot}}<10 \%\right)$ or higher $\left(P_{\mathrm{tot}}>20 \%\right)$ than average aggregated output can happen in any calendar day, however with much higher probability during the Summer (for lows) and Winter months (for highs), respectively. Note, however, that reliable output scheduling is already based on (shorttime) weather forecasts, because pure statistical methods are known to be very inaccurate. 


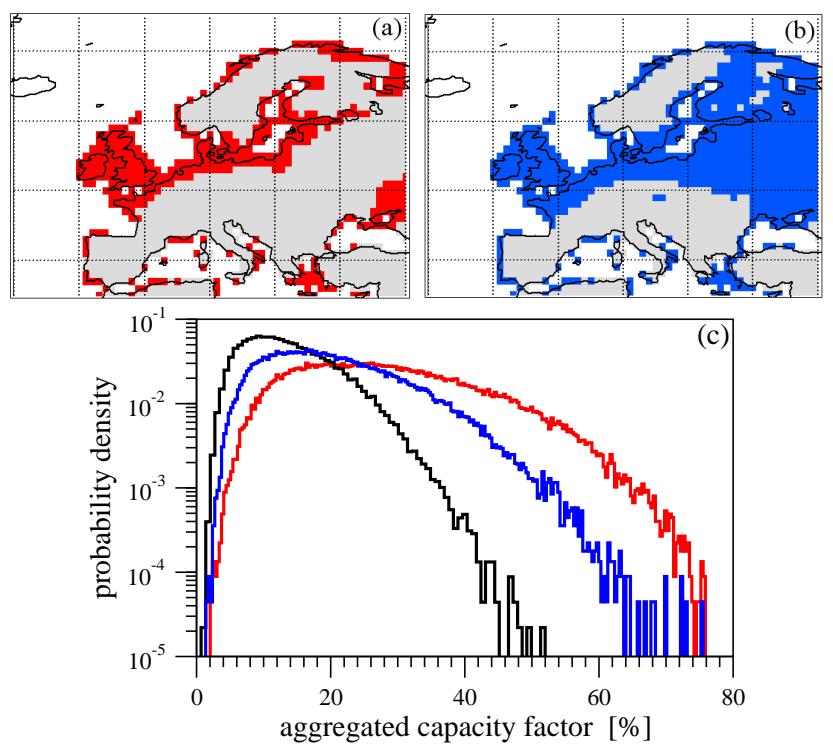

Fig. 7. (a) Geographic cells (424 out of the total 1325) where the 44 year average capacity factor is higher than $13.7 \%$, which is eqivalent to 1200 full load hours. (b) Geographic cells (765 out of 1325 ) where the 44 year average wind speed (at $10 \mathrm{~m}$ ) is higher than $3.5 \mathrm{~m} / \mathrm{s}$. (c) Normalised histograms of the aggregated capacity factor for three static network configurations. Black line: whole continent (the same as in Fig. 5b). Red line: network shown in (a). Blue line: network shown in (b).

Considering the apparent suboptimality of a whole continent network, other configurations might be also used as a reference for further comparisons. In Fig. 7 two examples are shown, where many low-wind sites are omitted by different threshold conditions. Figure 7a illustrates the geographic cells where the average capacity factor (over the 44 years) exceeds the threshold value of $13.7 \%$, which is equivalent with a minimum energy production of 1200 full load hours. Figure $7 \mathrm{~b}$ depicts a configuration where the threshold condition is given by the long time average wind speed at $10 \mathrm{~m}$ $(\langle s\rangle>3.5 \mathrm{~m} / \mathrm{s})$. Both networks produce aggregated output time series which are very similar to the whole continent reference case (Fig. 5a) with strong annual seasonality and a weak daily cycle (Fig. 5c). The marked differences are the increased average value and standard deviation apparent in Fig. 7c. (Numerical values are $29.7 \pm 13.1 \%$ for Fig. 7 a, and $21.5 \pm 10.1 \%$ for Fig. $7 \mathrm{~b}$.) The main problem with such networks is that the threshold conditions are pretty arbitrary, a small change in the numerical values can result in quite different configurations with different average values and standard deviation. That is the reason why we keep the whole continent integrated case for further reference.
3.2 Whole continent integration with optimised capacities

An obvious strategy to decrease the variability of aggregated output might be based on distributing available capacities (weights) unevenly. This could provide an effective way to harness temporal anticorrelations for different regions, therefore we determined the linear correlation coefficients

$\varrho_{i, j}=\frac{\left\langle\left[P_{i}(t)-\bar{P}_{i}\right]\left[P_{j}(t)-\bar{P}_{j}\right]\right\rangle}{\sigma_{i} \sigma_{j}}$

for all possible $(i, j)$ pairs of geographic cells, where $\bar{P}_{i}$ and $\sigma_{i}$ denote the mean and standard deviation in cell $i$, and $\langle\cdot\rangle$ indicates temporal averaging. Small (statistically non-significant) negative values for $\varrho_{i, j}$ are obtained for a few distant pairs, nevertheless we attempted to find uneven weight distributions with a decreased resulting variability of the aggregated output.

The procedure commences by assigning initial weight parameters $w_{i}$ to each geographic gridpoint $i$, representing the level of installed capacity at the given cell. In order to compare different configurations, normalisation is needed: $\sum_{i} w_{i}=1$, where $i=1, \ldots 1325$ (the total capacity is fixed), consequently $0 \leq w_{i} \leq 1$. (The uniform setting $w_{i}=1 / 1325$ reproduces the results discussed above.) The optimisation task is to modify the set $w_{i}$ in order to get a less variable aggregated output. This problem is a linearly constrained global optimisation in 1325 dimensions (Pardalos et al., 2000). We implemented an iterative Monte Carlo algorithm, where in one step a randomly chosen weight changes stochastically. In order to fulfil the normalisation constraint, two other randomly selected weights are modified. If the resulting configuration provides a smaller coefficient of variation for the aggregated power time series, then the set $w_{i}$ is updated and the iteration proceeds. This algorithm does not guarantee to find the global optimum, nevertheless it is able to produce improvements even for the fully connected case. An example from the many similar arrangements of almost identical performance is shown in Fig. 8, where the initial configuration was the uniform weight distribution. The long time mean aggregated capacity factor mildly increased to $14.4 \%$ (from $13.9 \%$ ) with the standard deviation of $4.1 \%$ (from $6.6 \%$ ), which means an improvement for the coefficient of variation to 0.28 (from 0.47). In spite of this improvement, the configuration shown in Fig. 8 hardly represents a serious advance. The offered network is based on a few highly efficient off-shore sites at the Atlantic coast connected to huge idle capacities located mostly at extremely low-wind cells in the opposite corner of the continent, where weak temporal anticorrelations exist at all. We emphasise that the lack of significant anticorrelations over the European continent is the main limiting factor here, therefore we have not tested further optimisation methods in this direction. 


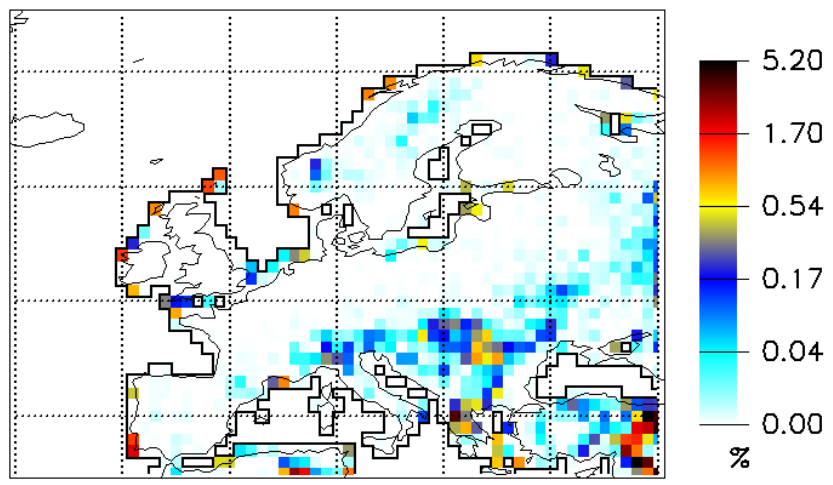

Fig. 8. Stochastically optimised global network configuration with different weight parameters for the cells. Percentage values of the final weights $\left(w_{i}\right)$ are indicated.

It is interesting to compare these results with a hypothetical static network of uneven weights based on existing installed capacities. The aggregated output of the total of 46.028 GW (October 2007) distributed in the appropriate geographic cells (see Fig. 9) has a 44-year mean value of $13.9 \%$ (the same as the whole-European uniform network above) with a much higher standard deviation of $12.0 \%$ (Fig. 10).

\subsection{Optimal limited area networks}

The suboptimal nature of whole-continent static integration is clearly demonstrated by evaluating aggregated time series over limited areas of high-wind locations (see Fig. 4a). As an example, Fig. 11 shows that the integrated wind power over the geographic cells around Great Britain obeys better statistics: the long time aggregated mean output is $39.7( \pm 25.1) \%$ and the probability distribution (Fig. 11b) has a much slower decay toward higher values than the whole-European reference case. Figure 11c compares an additional possible measure of quality, namely the probability density distribution for (percentage) ratio of sites at rated power. The rated power plateau (see Fig. 3) can be considered as the optimal operating range for turbines with respect to electric grid integration. The efficiency of electricity generation is lower here than at smaller wind speeds (a part of kinetic energy is lost during active control), but at least the output does not exaggerate the wind speed fluctuations in a strongly nonlinear way.

The geographic cells can be easily ranked by individually determining the temporal fraction of operation at rated power for the given period of 44 years. For example, the absolute majority of the 50 best geographic cells (hypothetical "base network") is around Great Britain and Ireland (see Fig. 12a). The long time mean value for such network is $52.1 \%$ with a standard deviation of $23.5 \%$. The annual periodicity remains quite strong, the absolute minimum value was obtained as $1.63 \%$ in the Summer of 1966. Nevertheless connections of distant locations

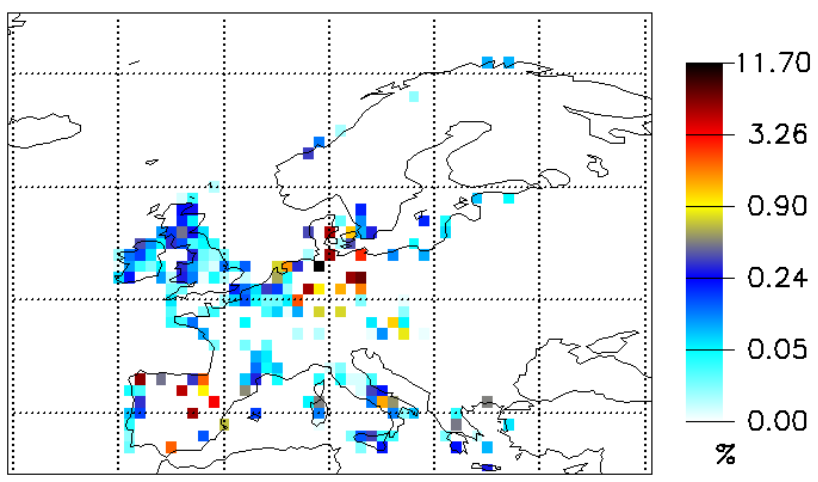

Fig. 9. Whole-continent hypothetical wind power network configuration based on installed capacities of October 2007. Weight factors $\left(w_{i}\right)$ in percentage of the total capacity of $46.028 \mathrm{GW}$ are coded in a logarithmic colour scale.

effectively eliminated the high probability of zero aggregated output, which is the case for the limited continuous area around Great Britain (see Fig. 11b).

As the numerical values of standard deviations or the histograms in Figs. 5b, 7b, 10b, 11b, or 12b indicate, a marked negative consequence of spatial integration over limited areas is an increased level of fluctuations around the mean aggregated output. The question naturally arises: how large improvement can we expect by gradually increasing the size of the network? One possible measure is illustrated in Fig. 13, where the test was constructed in the following way.

The simulated wind power time series for a given number $(n=1, \ldots 1324)$ of randomly chosen cells were aggregated at each time step, and the long term mean value $P_{\text {tot }}$ and its standard deviation $\sigma$ were computed, as before. At each network size $n, 30$ different random realisations were evaluated and ensemble averages $\left\langle P_{\text {tot }}\right\rangle$ and $\langle\sigma\rangle$ were determined. The results are plotted in Fig. 13, where the number $n$ was transformed to total network area with correcting Earth's surface curvature. Figure 13a illustrates that the ensemble averaged capacity factor is essentially independent of the network size, the average for 30 random single cells is practically the same as for the whole-scale integrated value. The behaviour of the ensemble averaged standard deviation shown in Fig. 13b is more remarkable. If the individual cell outputs represented statistically independent random variables, this parameter should decay according to an inverse square root law, illustrated by the grey dashed line in Fig. 13b. The empirical data indicate marked deviation from this expectation for network sizes of $15-17$ cells already.

This behaviour clearly implies the role of strong spatial correlations. The linear correlation coefficients given by Eq. (2) decay exponentially as a function of cell-cell distance $r_{i, j}$ :

$\varrho_{i, j}\left(r_{i, j}\right) \propto \exp \left(-r_{i, j} / L_{i}^{*}\right)$

where $L_{i}^{*}$ is the average correlation length for the cell $i$, 


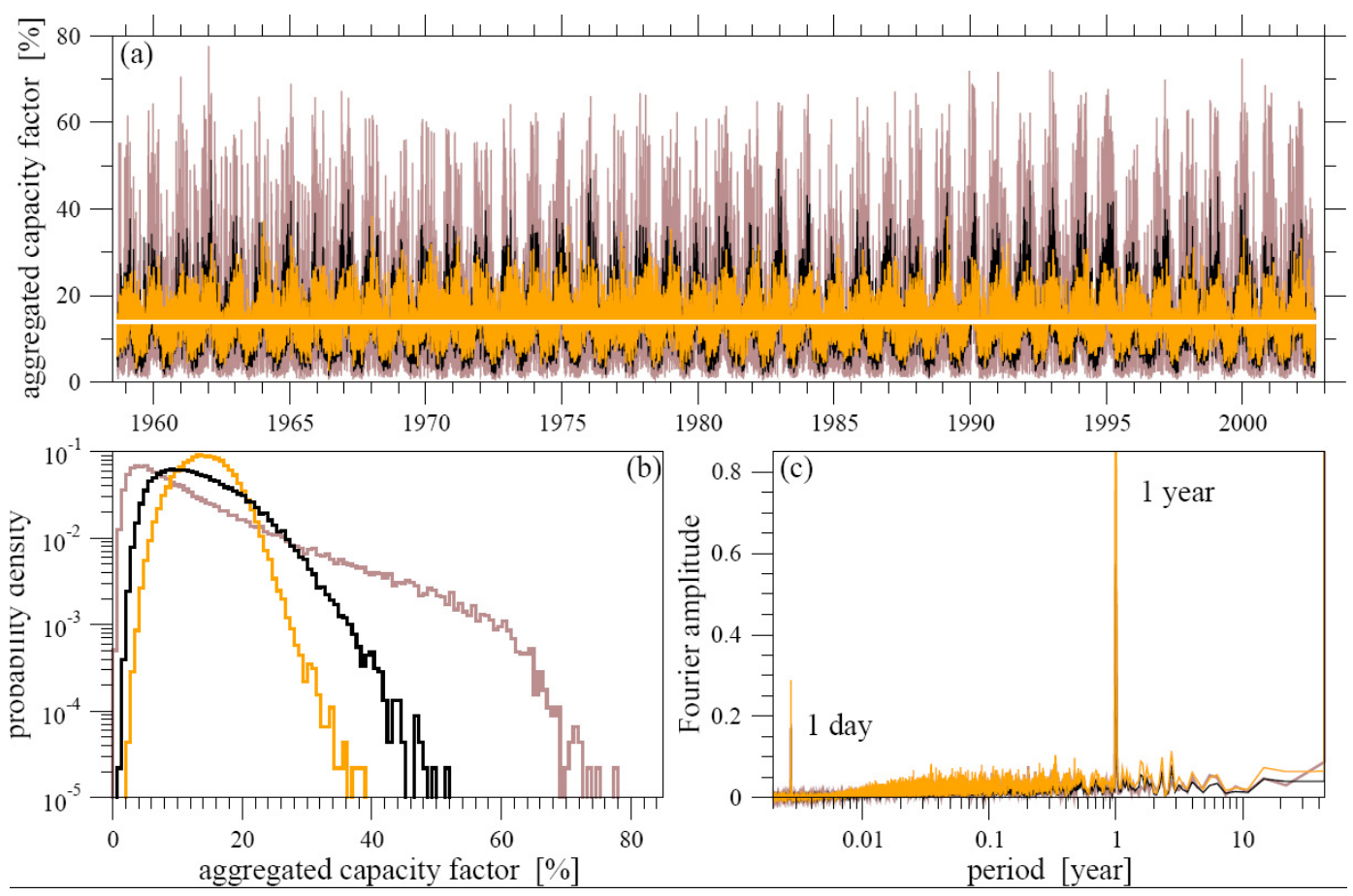

Fig. 10. Comparison of the aggregated output for the whole-European static network (black), for the network based on existing capacities (brown) and for the static network of optimised weights (orange). (a) Time series. (b) Probability density distribution of the aggregated output. The mean values are practically the same $(13.9 \%, 13.9 \%, 14.4 \%)$ with very different standard deviations $(6.6 \%, 12.0 \%, 4.1 \%)$. Note that the histograms are strongly non-Gaussian, therefore these numerical values have limited information. (c) Power density spectrum as a function of period.

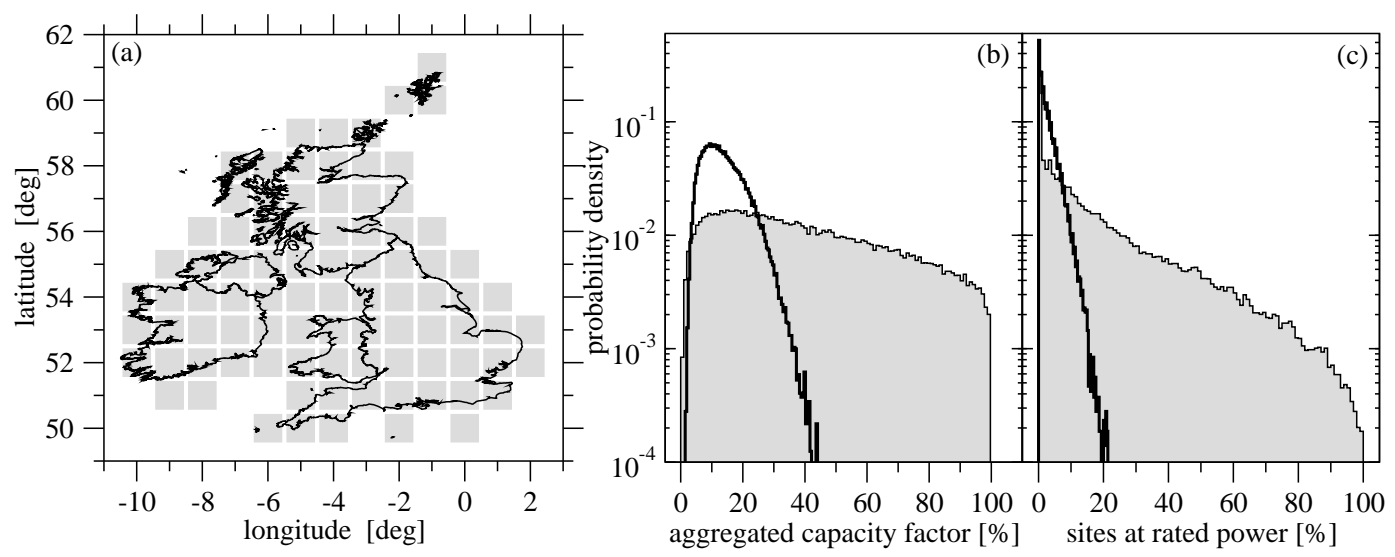

Fig. 11. (a) Geographic setting of the analysed area around Great Britain. (b) Probability density distribution for the aggregated capacity factor (grey histogram). Black line shows the whole-European curve in Fig. 5b. (c) Probability density distribution for the percentage ratio of sites at rated power (grey), the black curve is the same for the whole-European network.

averaged over all geographic directions. This smears out the existing anisotropies, however gives an approximate measure for the strength of spatial correlations. Typical values for $L_{i}^{*}$ are $400-600 \mathrm{~km}$ both over land and the ocean (see also Fig. 15a).

\subsection{Dynamically restructured networks}

Although all the basic statistical information about wind power availability is included in the whole-European integrated model time series characterised by Figs. 5 and 6, in the final test we demonstrate further serious practical limitations by using a fully connected network architecture 

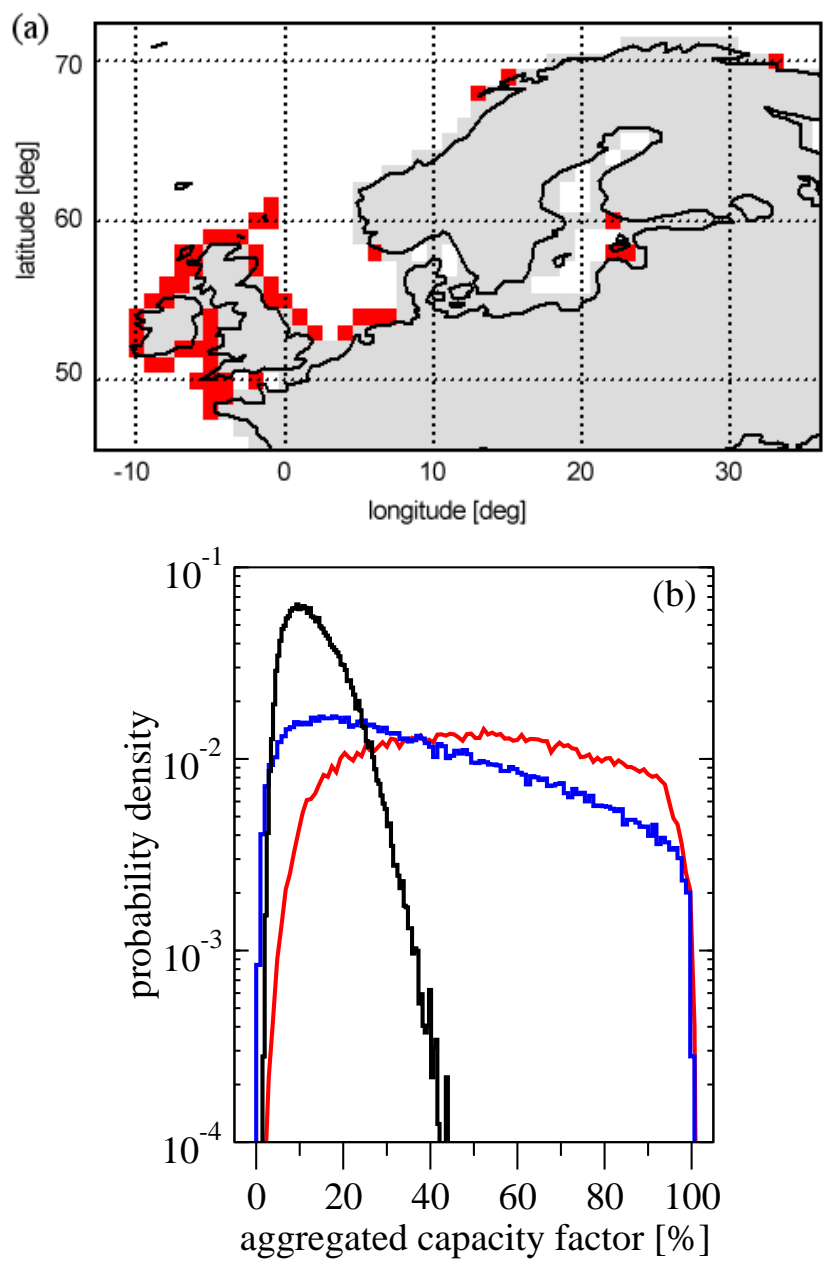

Fig. 12. (a)"Base network" formed by the 50 best wind sites over the continent (red cells). (b) Probability density distribution of the aggregated capacity factor for Great Britain (blue, Fig. 11a), for the base network shown in (a) (red), and for the whole continent (black curve, Fig. 5b).

with dynamic control. The target is to achieve a smooth aggregated power generation at a modest level corresponding to the maximal integrated power of 50 cells out of the available 1325 .

The feature of dynamic control means that at a given time instant any number of cells can be connected to produce the target output, irrespective of the geographic locations. When the output of a given operating site drops, the missing power is replaced by connecting another site(s) to the grid. In the simulations, the dynamic rewiring is based on a list where the cells are ranked by the level of instantaneous potential output. The main question of this analysis is the number of sites necessary to produce the target output.

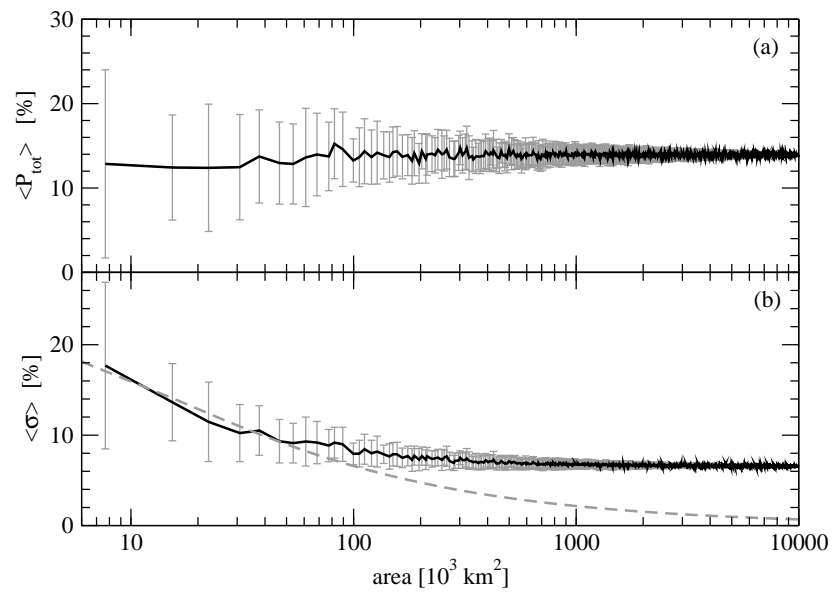

Fig. 13. Ensemble and time averaged aggregated capacity factor $\left\langle P_{\text {tot }}\right\rangle$ for 30 samples of randomly connected cells of increasing number. The number of connected cells is transformed to total integrated area on the horizontal axes. The error bars represent standard deviation computed for the 30 realisations. (b) Averaged standard deviation $\langle\sigma\rangle$ around the mean $\left\langle P_{\text {tot }}\right\rangle$ for the same test. The grey dashed line illustrates an inverse square root function.

The results in Fig. 14 demonstrate that even such a low target level cannot be sustained without serious breaks. It happens regularly during the Summer months that the aggregated output of all the available cells is less than the required power. Figure $14 \mathrm{~b}$ illustrates that the periodically supervening global low-wind situation is characterised also by the extremely high number of cells (close to 33\%) where the wind speed remains below the cut-in value.

This behaviour is partly the consequence of very limited temporal correlations of wind speeds. The autocorrelation functions $A_{i}(\tau)$ decay also exponentially

$A_{i}(\tau)=\frac{\left\langle\left[P_{i}(t)-\bar{P}_{i}\right]\left[P_{i}(t+\tau)-\bar{P}_{i}\right]\right\rangle}{\sigma_{i}^{2}} \propto \exp \left(-\frac{\tau}{T_{i}^{*}}\right)$

where the notations are the same as before. Typical values for the correlation time $T_{i}^{*}$ hardly exceed 2 days (see also Fig. 15b), which is also reflected by the very dynamic rewiring of the integration network in the test.

\section{Summary and conclusions}

The main conclusions of our model studies are the following. (i) Whole-scale static integration of wind electricity over the continental Europe produces an output of low average capacity factor and marked annual seasonality. (ii) Weak anticorrelations exist for very distant geographic locations, but the fluctuations of the integrated power can be decreased only at a low level of average output. (iii) Integration over limited geographic areas can result in higher average capacity factors at the expense of stronger fluctuations. (iv) The strong spatial correlations present in the wind field determine a lower 


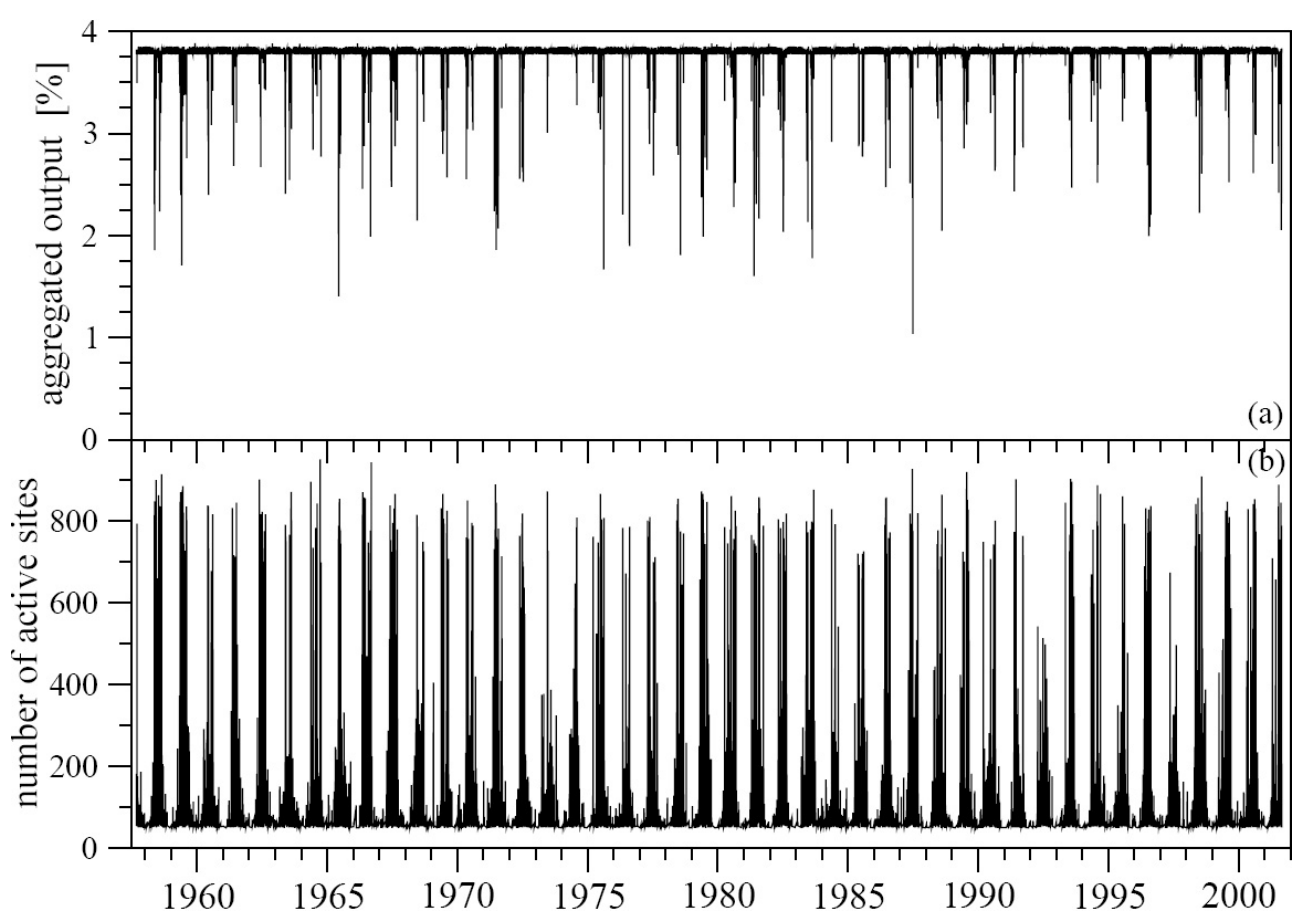

Fig. 14. (a) Aggregated output from a dynamically controlled, fully integrated grid expressed as percentage ratio of the total installed capacity. The target level is 50 cells at rated power (3.77\%). (b) Number of simultaneously operating cells.

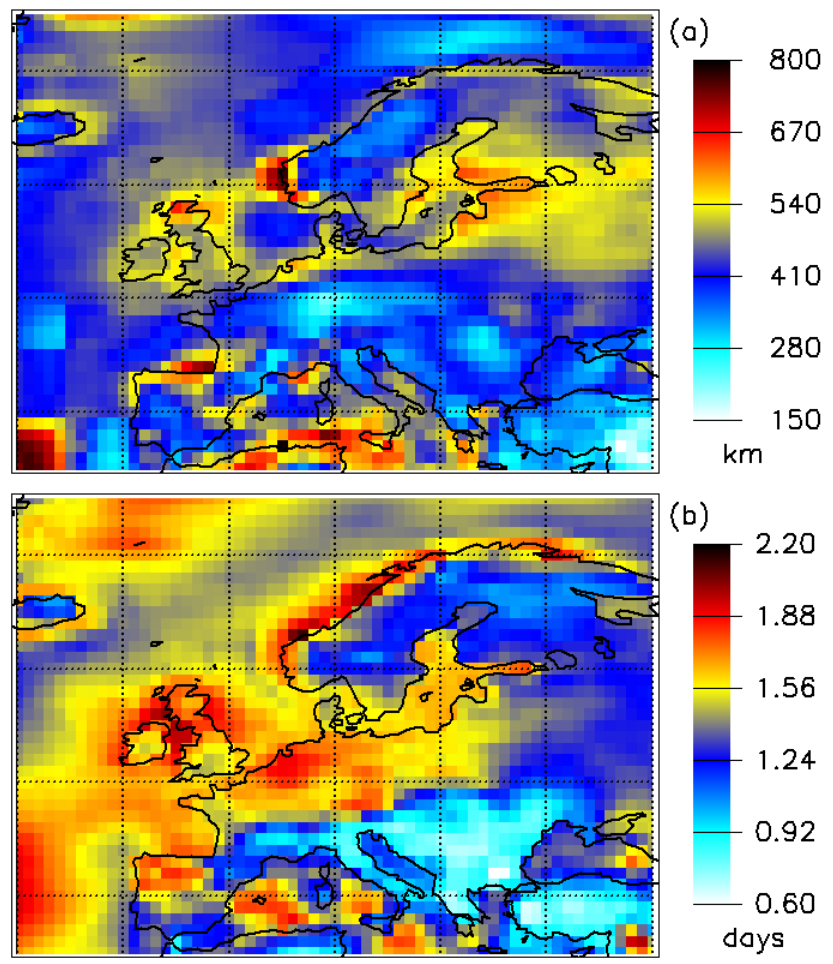

Fig. 15. (a) Average correlation length $L^{*}$ (see Eq. 3) and (b) autocorrelation time $T^{*}$ (see Eq. 4) for each geographic cell obtained from the whole period of 44 years. limit for the fluctuations of aggregated outputs which can not be eliminated by increasing the area of electricity integration. (v) Dynamic control can produce smooth aggregated output at low average levels, however the effects of periodically occurring global low-wind situations cannot be eliminated.

We emphasise that our conclusions are completely based on the wind field records provided by the ERA-40 data bank. We are fully aware of the limited temporal and spatial resolution of the time series, nevertheless we think that the most essential information about wind climatology is incorporated. The simple method we implemented for wind speed estimates at higher elevations can be a source of numerical errors. Also average values are sensitive to the prefactor used in the computations, but the main conclusion on the repeatedly occurring global low-wind situations remain unaltered.

To avoid any misunderstanding, we would like to emphasise again that our results have nothing to do with the utilisation of wind energy itself. In the contrary, we are convinced that the present civilisation is unsustainable where $80 \%$ of primary energy comes from oil, coal, and natural gas combined. On the other hand, we also think that the frequent allusion of the tiny fraction of incoming solar radiation covering the global human energy consumption conveys a somewhat misleading message to the public. It might suggest that an easy switch to renewables is possible with the present day technologies, and this is a mere question of political decision and some moderate investment; but we are not so optimistic in such conclusions. 
Acknowledgements. We thank the Hungarian Meteorological Service for the access to the ERA-40 data bank. This work was supported by the European Commission's DG RTD NEST Programme "Tackling Complexity in Science" (contract No. 043363), and by the Hungarian Science Foundation (OTKA) under Grant No. NK72037. IMJ thanks for a János Bolyai research scholarship of the Hungarian Academy of Sciences.

Edited by: A. Baas

Reviewed by: two anonymous referees

\section{References}

Ackermann, T. and Söder, L.: An overview of wind energy-status 2002, Renew. Sust. Energ. Rev., 6, 67-127, 2002.

Archer, C. L. and Jacobson, M. Z.: Evaluation of global wind power, J. Geophys. Res., 110, D12110, doi:10.1029/2004JD005462, 2005.

Burton, T., Sharpe, D., Jenkins, N., and Bossanyi, E.: Wind Energy Handbook, John Wiley \& Sons, Chichester, 648 pp., 2001.

Carl, D. M., Tarbell, T. C., and Panofsky, H. A.: Profiles of wind and temperature from towers over homogeneous terrain, J. Atmos. Sci., 30, 788-794, 1973.

Dondi, P., Bayoumib, D., Haederlic, C., Julian, D., and Suter, M.: Network integration of distributed power generation, J. Power Sources, 106, 1-9, 2002.

Elliott, D. L., Holladay, C. G., Barchet, W. R., Foote, H. P., and Sandusky, W. F.: Wind Energy Resource Atlas of the United States, US Department of Energy, (http://rredc.nrel.gov/wind/ pubs/atlas/) (last access: 28 September 2008), 1986.

European Wind Energy Association: Wind Energy - The Facts, (http://www.ewea.org) (last access: 28 September 2008), 2004.

Farrugia, R. N.: The wind shear exponent in a Mediterranean island climate, Renew. Energ., 28, 647-653, 2003.

Garratt, J. R.: The Atmospheric Boundary Layer, Cambridge University Press, Cambridge, 340 pp., 1994.

Giebel, G.: A variance analysis of the capacity displaced by wind energy in Europe, Wind Energy, 10, 69-79, 2007.

Henderson, A. R., Morgan, C., Smith, B., Srensen, H. C., Barthelmie, R. J., and Boesmans, B.: Offshore wind energy in Europe - A review of the state-of-the-art, Wind Energy, 6, 3552, 2003.
Holttinen, H.: Hourly wind power variations in the Nordic countries, Wind Energy, 8, 173-195, 2005.

Hsu, S. A., Meindl, E. A., and Gilhousen, D. B.: Determining the power-law wind-profile exponent under near-neutral stability conditions at sea, J. Appl. Meteorol., 33, 757-765, 1994.

Katsaprakakis, D. A., Papadakis, N., Christakis, D. G., and Zervos, A.: On the wind power rejection in the islands of Crete and Rhodes, Wind Energy, 10, 415-434, 2007.

Kiss, P. and Jánosi, I. M.: Comprehensive empirical analysis of ERA-40 surface wind speed distribution over Europe, Energ. Convers. Manage., 49, 2142-2151, 2008.

Landberg, L.: The availability and variability of the European wind resource, International Journal of Sustainable Energy, 18, 313320, 1997.

Lund, H.: Large-scale integration of wind power into different energy systems, Energy, 30, 2402-2412, 2005.

Pardalos, P. M., Romeijn, H. E., and Tuy, H.: Recent developments and trends in global optimization, J. Comput. Appl. Math., 124, 209-228, 2000.

Papaefthymiou, G., Schavemaker, P. H., van der Sluis, L., Kling, W. L., Kurowicka, D., and Cooke, R. M.: Integration of stochastic generation in power systems, Int. J. Elec. Power, 28, 655-667, (2006).

Pepermans, G., Driesen, J., Haeseldonckx, D., Belmans, R., and Dhaeseleer, W.: Distributed generation: definition, benefits and issues, Energy Policy, 33, 787-798, 2005.

Pryor, S. C. and Barthelmie, R. J.: Comparison of potential power production at on- and offshore sites, Wind Energy, 4, 173-181, 2001.

Sedefian, L.: On the vertical extrapolation of mean wind power density, J. Appl. Meteorol., 19, 488-493, 1980.

Tennekes, H.: The logarithmic wind profile, J. Atmos. Sci., 30, 234-238, 1973.

Troen, I. and Petersen, E. L.: European Wind Atlas, (Risoe National Laboratory, Roskilde), 656 pp., 1989.

Uppala, S. M., Kallberg, P. W., Simmons, A. J., et al.: The ERA-40 re-analysis, Q. J. Roy. Meteor. Soc., 131, 2961-3012, 2005. 